\title{
ORIGINAL
}

\section{PARTICIPACIÓN SOCIAL EN SALUD: CONCEPTOS DE USUARIOS, LÍDERES COMUNITARIOS, GESTORES Y FORMULADORES DE POLÍTICAS EN COLOMBIA. UNA MIRADA CUALITATIVA $(*)$}

María Eugenia Delgado Gallego (1), María Luisa Vázquez Navarrete (2), Yolanda Zapata Bermúdez (3) y Mariano Hernán García (4)

(1) GEMA- Universidad del Valle, Colombia.

(2) Consorci Hospitalari de Catalunya.

(3) Universidad del Valle. Colombia.

(4) Escuela Andaluza de Salud Pública.

\section{RESUMEN}

Fundamento: Las reformas del sector salud llevadas a cabo en Colombia durante la década de los noventa incluyen políticas que promocionan la participación social en el sistema de salud, considerada esencial para el buen funcionamiento. El objetivo de este artículo es analizar el sentido y significado de la participación en salud para los diferentes actores sociales involucrados en la implantación de la política en Colombia.

Métodos: Se realizó un estudio cualitativo, descriptivo, de tipo exploratorio, a través de grupos focales (GF) y entrevistas individuales semi-estructuradas (E) a diferentes actores sociales: 210 usuarios (GF), 40 líderes comunitarios (GF), 3 formuladores de políticas (E) y 36 entre el personal de salud (E). Se hizo un análisis narrativo de contenido. El área de estudio la constituyeron los municipios Tuluá y Palmira en Colombia.

Resultados: El concepto de participación presentó diferentes sentidos según el actor analizado: usuarios y líderes articulaban el concepto alrededor de aportar ideas, estar en espacios sociales, la solidaridad y con frecuencia, la utilización de los servicios de salud. El daridad y con frecuencia, la utilización de los servicios de salud. El
personal de salud otorgaba sentido de participación social a las activipersonal de salud otorgaba sentido de participación social a las activi-
dades de las instituciones con la comunidad, así como al uso de los servicios y la vinculación al sistema de salud. Los formuladores de políticas lo dotaban de un sentido de evaluación y control de los servicios de salud por parte de la comunidad, para mejorar su calidad.

Conclusiones: Los distintos conceptos de participación muestran diferencias entre el sentido de la política y lo que entienden y asumen los diversos agentes sociales en su interacción con los servicios de salud. Estos diferentes conceptos han de ser tenidos en cuenta para conseguir la vinculación entre la sociedad y los servicios de salud.

Palabras clave: Participación en salud. Investigación cualitativa. Colombia.

Correspondencia:

María Eugenia Delgado Gallego

Universidad del Valle. Instituto de Psicología. Edificio 388.

Calle 13\#100-00. Sede Meléndez.

Cali, Valle. Colombia

Correo electrónico: mariedel@ andinet.com

\section{ABSTRACT}

\section{Social Participation in Health: User \\ Community Leaders, Managers and Policy Makes \\ in Colombia. A Qualitative View}

Background: Health sector reforms taking place in Colombia during the Nineties included policies to promote social participation in the health system, which is considered essential to its functioning. The aim of this article is to analyse the meaning and the sigtioning. The aim of this article is to analyse the meaning and the sig-
nificance of participation in health for the different social actors involved in implementing policies in Colombia.

Methods: A qualitative, descriptive, exploratory study was carried out using focal groups (FG) and semi-structured individual interviews (I) of the different social actors: 210 users (FG), 40 community leaders (FG), 3 policy makers (E) and 36 healthcare professionals (E). A carried out analysis was content up of the contents. The study area corresponded to the municipalities of Tulua and Palmira in Colombia.

Results: The concept of participation was interpreted differently depending on the actor studied: for users and leaders the concept referred to contributing ideas, presence in social spaces, solidarity and frequently, and use of the health services. Healthcare professionals considered the activities carried out by institutions together with the community as social participation, the use of services and affiliation to the health system. Policy markers considered participaaffiliation to the health system. Policy markers considered participa-
tion to concern evaluation and control of the health services by the community, to improve its quality.

Conclusions: The different concepts of participation reveal differences between the content of the policy and how it is understood and interpreted by the different social actors in their interaction with the health services. These different perspectives must be taken into account to develop a link between society and the health services.

(*) Financiación de la Comisión Europea. DG XXII (Contrato: IC18-CT98-0340) 


\section{INTRODUCCIÓN}

La reforma del sector salud en Colombia, que crea el Sistema General de Seguridad Social en Salud (SGSSS), proporciona un nuevo impulso a la participación social en salud, que se señala como elemento fundamental en la organización y control de las instituciones del Sistema de Salud (Constitución del 1991, ley 100 del 1993, decreto 1757/1994). En este nuevo marco, la participación en salud se define como derecho y deber del individuo y se instituye una serie de mecanismos de uso individual como buzón de quejas, línea telefónica, oficina de atención al usuarios (OAU); así como se establece una serie de mecanismos institucionales para la participación colectiva, como Asociaciones de Usuarios (AU), los Comités de Ética Hospitalaria, Comités de Veeduría, representantes de las AU en las Juntas Directivas de Instituciones Prestadoras de Servicio (IPS) y de las Entidades Promotoras de Salud (EPS) públicas ${ }^{1}$.

En el marco legal, por tanto, la participación social se constituye en estrategia para la producción social de la salud a partir de la interacción de los diversos actores, que construyen diferentes significados de forma conjunta, y adquiere un sentido político ya que otorga poder a los que antes carecían de él. No obstante, se ha estudiado muy poco la percepción de la participación de los distintos actores que intervienen en el proce$\mathrm{so}^{2,3,4,5}$. Casi todos los trabajos sobre participación en salud se ubican en el estudio crítico de los procesos de implantación de la estrategia en programas de salud $d^{6,7,8,9}$, o con relación a experiencias concretas de la participación en proyectos de control de endemias o de promoción de la salud ${ }^{10,11}$, o trabajos dirigidos a elaborar una sistematización sobre formas y niveles de participación y propuestas para evaluarla ${ }^{12,13,9}$. La gran escasez de estudios hace necesario el desarrollo de trabajos donde se considere entre otras, una dimensión intersubjetiva, es decir, el análisis de factores psicosociales determi- nantes en la implantación de políticas de participación en salud, como las percepciones, representaciones y opiniones que configuran el universo simbólico construido alrededor del concepto de participación por los actores involucrados en el proceso $0^{5,14,15}$. Los distintos actores sociales, como individuos o grupos, interactúan cotidianamente, entre otros, en el marco de consolidación de la política de participación, situación que de acuerdo al interaccionismo simbólico ${ }^{28}$ los lleva a configurarse mutuamente.

Considerando lo anterior, se desarrolló una investigación amplia con el objeto de evaluar la efectividad de la actual política de promoción de la participación de los usuarios en los servicios de salud, dentro de la reforma del sector salud en Colombia. Se analizaron diversos aspectos relativos al contenido de la política, implementación de los mecanismos de participación, opiniones, conocimiento y utilización por los diferentes actores sociales. El objetivo del presente artículo es analizar las ideas, significados y sentidos construidos de los conceptos de participación social en salud, por los principales actores sociales involucrados en la implantación de la política de participación en el control de la calidad de los servicios de salud en Colombia.

\section{Aspectos conceptuales sobre participación social}

El concepto de participación social es interpretado y definido de múltiples maneras y por lo tanto no está libre de contradicciones, incoherencias, ambigüedades ${ }^{16}$, polémicas e imprecisiones de carácter teórico ${ }^{6}$. La participación es un proceso dinámico, oscilante que se puede definir de acuerdo a las características del contexto social y político, así como del momento en que se reali$\mathrm{za}^{4}$, y adquiere diferentes sentidos si se la considera como fin en si misma, o como medio para lograr ciertas metas 9 . También se podría decir que los diferentes niveles de 
participación prefiguran el sentido que los actores involucrados le dan a la participación. Estos niveles son, partiendo del más bajo al de mayor grado: información, consulta facultativa, elaboración y recomendación de propuestas (iniciativa), fiscalización, concertación, co-gestión y toma de decisiones, y la autogestión ${ }^{17,18}$. Además, la participación social ha sido considerada como norma para realizar una tarea conjunta ${ }^{19}$; como esfuerzo organizado para incrementar el control sobre los recursos y las instituciones reguladoras en situaciones sociales dadas ${ }^{20,21}$; como «empoderamiento» de la comunidad local mediante un proceso a través del cual grupos de población que carecen de acceso equitativo a recursos valorados, ganan control (poder) sobre esos recursos. La participación como empoderamiento implica interacción, respeto mutuo, reflexión crítica, acción grupal, compromiso, influencia e información, y por tanto, para poder participar efectivamente se requiere acceso real a la educación, a la información, a las actividades sociales y políticas y a la tecnología ${ }^{22}$. La participación también se entiende como actitud de solidaridad, de diálogo y preocupación por la gestión de la vida colectiva. Actitud democrática de quien gobierna, que permita escuchar a los ciudadanos, darles protagonismo en la vida de las instituciones democráticas ${ }^{23}$. Además, la participación se define como actividad organizada por parte de un grupo, con el objeto de expresar necesidades, demandas, de defender intereses comunes, de influir de manera directa en los poderes públicos ganando así poder político ${ }^{24}$. Siendo muchas las definiciones teóricas del concepto, todas poseen en común el reconocimiento del control político que se puede alcanzar si se participa de manera autónoma, conciente y $\operatorname{organizada}^{38}$.

\section{SUJETOS Y MÉTODOS}

Área de estudio. El estudio se llevó a cabo en dos municipios del Departamento del Valle del Cauca, sur occidente de Colombia: Tuluá, con 184.725 habitantes y donde la reforma del sector salud se inició en 1996; y Palmira, con 283.431 habitantes, cuya reforma comenzó en 1993.

Los municipios se seleccionaron aplicando los siguientes criterios: haber implementado la reforma del sector salud; poseer población de todos los estratos socioeconómicos; contar con porcentajes altos de afiliación al sistema de seguridad social en salud; disponer de infraestructura en todos los niveles de atención y un municipio con carácter más rural y otro más urbano.

Métodos. Se realizó un estudio cualitativo utilizando entrevistas individuales semiestructuradas (E) y grupos focales (GF), para recoger los conceptos y opiniones acerca de la participación en salud de los diferentes actores sociales implicados en la implantación de la política. Las guías de entrevistas individuales (al personal de salud y formuladores de políticas) y de los grupos focales (a usuarios y líderes) poseían una parte común y otra específica según el tipo de entrevistado. Tanto las entrevistas como los grupos focales fueron grabados y transcritos textualmente. La duración de las entrevistas y grupos focales fue de 60 a 90 minutos. El trabajo de campo se realizó entre el 2000 y el 2001 .

Muestra. Se diseñó una muestra teórica que abarcaba los diversos actores sociales que pueden afectar o verse afectados por la política. La composición final de la muestra fue: 210 usuarios (80 en Palmira, 130 en Tuluá; mediante $20 \mathrm{GF}$ ); 40 líderes comunitarios (22 Palmira y 18 en Tulúa; mediante 5 GF); 36 del personal de salud (13 gerentes en Palmira y 13 gerentes en Tulúa; 4 encargados de las Oficinas de Atención al Usuario en Palmira y 6 en Tulúa), y 3 formuladores de políticas de nivel regional y municipal (Tabla 1). La muestra de usuarios se seleccionó de forma intencional para lograr la máxima variación siguiendo criterios de 
Tabla 1

Composición final de la muestra

\begin{tabular}{|c|c|c|c|c|c|}
\hline \multirow[b]{2}{*}{$\begin{array}{c}\text { Tipo de } \\
\text { Actor social }\end{array}$} & \multicolumn{2}{|c|}{ Palmira } & \multicolumn{2}{|c|}{ Tulúa } & \multirow{2}{*}{$\begin{array}{l}\text { Regional } \\
\text { Entrevista } \\
\text { Individual }\end{array}$} \\
\hline & $\begin{array}{l}\text { Grupo } \\
\text { Focal }\end{array}$ & $\begin{array}{l}\text { Entrevista } \\
\text { Individual }\end{array}$ & $\begin{array}{l}\text { Grupo } \\
\text { Focal }\end{array}$ & $\begin{array}{l}\text { Entrevista } \\
\text { Individual }\end{array}$ & \\
\hline Usuarios & $10(80)$ & & $10(130)$ & & \\
\hline Líderes & 3 (22) & & $2(18)$ & & \\
\hline Gerentes (IPS- EPS) & & 13 & & 13 & \\
\hline $\begin{array}{l}\text { Encargado Oficina } \\
\text { Atención al Usuario }\end{array}$ & & 4 & & 6 & \\
\hline $\begin{array}{l}\text { Secretario Salud } \\
\text { Municipal }\end{array}$ & & 1 & & 1 & \\
\hline $\begin{array}{l}\text { Secretario Salud } \\
\text { Departamental }\end{array}$ & & & & & 1 \\
\hline
\end{tabular}

edad (jóvenes: de 18 a 35 años; adultos de 36 a 55 años, mayores: mayores de 55 años), tipo de afiliación a la SGSSS (vinculados, subsidiados, contributivos), estrato social (estrato bajo: 1-2; medio: 3-4; alto: 5-6) y sexo (hombres y mujeres). Se definió como líderes a los representantes de los usuarios en las ligas de usuarios de las IPS y representantes de la comunidad ante las Juntas de Acción Comunal (JAC). El personal de salud se seleccionó teniendo en cuenta la titularidad (pública/privada), el tipo institución (aseguradoras, EPS/ARS; institución prestadora del servicio de salud, IPS), y categoría laboral (Gerentes y encargado de OAU).

Análisis y calidad de los datos. Se realizó un análisis de contenido narrativo, siguiendo un proceso de etapas diferenciadas: 1) segmentación de los datos por municipio y por actores. 2) Generación mixta de categorías, mediante la decantación de los contenidos en matrices de análisis, a) primero a partir de las categorías que conformaron los guiones de las entrevistas y de los GF y b) a partir de la lectura cuidadosa de los datos así ordenados, se detectaron categorías emergentes o unidades de sentido que surgieron de los discursos. Se elaboraron las matrices y consolidados con ayuda del procesador de palabras. Para garantizar la calidad de los datos se contrastaron los datos con los entrevistados al final de la entrevista, se realizaron descripciones amplias y se llevó a cabo la triangulación ${ }^{25}$ a partir de diferentes fuentes de datos y lugares, diferentes investigadores en la recogida y análisis de datos, diferentes técnicas; y la auditoria por un investigador externo.

\section{RESULTADOS}

\section{Concepto de participación social en salud de usuarios y líderes}

Se observó en las respuestas de los usuarios y líderes cierta dificultad para definir el concepto de participación social en salud, más bien recurrieron a diversas imágenes, ideas y actividades concretas en la vida cotidiana. En el discurso de los usuarios y líderes entrevistados, sus conocimientos y conceptos de participación en salud se aglutinan y ordenan alrededor de 6 unidades de sentido 
Tabla 2

Conceptos de participación en salud según tipo de actor social

\begin{tabular}{|c|c|}
\hline Actor Social & Concepto de participación en salud \\
\hline Usuarios y líderes & $\begin{array}{l}\text { - Participación social en salud como dar o aportar ideas y opiniones. } \\
\text { - Participación social en salud como colaboración. } \\
\text { - Participación social en salud como utilización de los servicios de salud y vinculación al sistema de } \\
\text { salud: } \\
\text { - Participación social en salud como derecho y } \\
\text { - Participación social como capacidad (sólo líderes) } \\
\text {-Participación como organización comunitaria }\end{array}$ \\
\hline Personal de salud & $\begin{array}{l}\text {-Participación social en salud como actividades con la comunidad (promoción, prevención y } \\
\text { ejecución de algunos programas) } \\
\text {-Participación en salud como forma de control y seguimiento a los servicios de salud. } \\
\text {-Participación social en salud como implicación en procesos institucionales } \\
\text {-Participación en salud como estar afiliado al sistema de salud. }\end{array}$ \\
\hline $\begin{array}{l}\text { Formuladores de } \\
\text { políticas }\end{array}$ & $\begin{array}{l}\text {-Participación en salud como evaluación y control social de los servicios de salud por parte de los } \\
\text { usuarios. }\end{array}$ \\
\hline
\end{tabular}

sobresalientes: aportar ideas; colaborar; pertenecer al sistema y usar los servicios de salud; derecho; capacidad; y organización comunitaria. Mientras que las tres primeras son compartidas por todos los informantes, las tres últimas emergieron fundamentalmente del discurso de los líderes comunitarios.

Participación como aportar opiniones a las instituciones de salud. El concepto de participación en salud para muchos de los usuarios se relaciona con la noción de aportar ideas y opiniones, espontáneamente o a través de las encuestas que realizan las instituciones de salud. Esta idea atravesó el discurso de la mayoría de los entrevistados. "Darle uno opiniones a la entidad de salud para que traten de mejorar el servicio». (G.F 35-50 años Tuluá).

Participación como colaboración. Para algunos entrevistados, sobre todo de los estratos bajos, la participación en salud tiene un sentido de ayuda y colaboración con los demás: «Participar en salud es colaborar con la gente de la comunidad.....orientarla en los servicios de salud» (G.F 35-50 años Palmira).
Participación como afiliación al sistema y utilización de los servicios de salud. La mayoría de los usuarios jóvenes y algunos usuarios mayores de 50 años describían la participación en salud como un conjunto de acciones muy concretas que se circunscriben al ámbito del uso de los servicios de salud: «En el caso personal la única vez que uno participa, es cuando uno se inscribe $y$ cuando se enferma» (G.F Mayores de 50 años Palmira). "Participar es de vez en cuando ir al médico» (G.F 18-35 años Tuluá). Igualmente algunos usuarios de estrato alto y medio alto consideraban que participar en salud era pertenecer al sistema de salud. «.. es estar vinculado a la empresa, a la EPS, al Seguro, a lo que sea» (G.F 1835 años Tulúa).

Participación como derecho. Solamente de las manifestaciones de los líderes, emergió el sentido de participación social en salud como el derecho de estar presentes en las actividades y de recibir apoyo para un bien colectivo. «...es el derecho de participar en actividades que se lleven a cabo en la misma comunidad para el bien de todos» (Líder Palmira). 
Participación como capacidad. Así mismo, los líderes en las asociaciones de usuarios entendían la participación social en salud como la capacidad de la comunidad para actuar sobre aspectos relacionados con la prestación de los servicios de salud: «Capacidad para influir en la calidad de los servicios de salud, capacidad para informar a los demás lo que pasa, capacidad para expresar las decisiones» (Representante de la A.U Tulúa).

Participación como organización comunitaria. Varios líderes mencionaron la importancia de la organización comunitaria y del trabajo colectivo como núcleo significativo de su concepto de participación en salud: "Participar es que hay que saber organizarnos para pedir, es importante la forma que vamos a pedir nuestro derecho, porque lo primero que todo, si pedimos mal o si no pedimos todos en comunidad, nos desvían todos los recursos, en este momento la salud la tienen olvidada y es por eso...» (Líderes Palmira).

\section{Concepto de participación social en salud del personal de salud}

De manera semejante al grupo de usuarios y líderes, la definición del concepto de participación en salud entrañó una dificultad para la mayoría de los entrevistados del grupo de personal de salud (gerentes y encargados de OAU). Así, proporcionaron una variedad de definiciones que remitían casi siempre a ideas concretas de interacciones sociales entre la institución y la comunidad o a situaciones sociales cotidianas. Del discurso de estos actores emergieron cuatro unidades de sentido alrededor de las cuales se ordenan sus conocimientos y conceptos acerca de la participación en salud. Estos núcleos o unidades de sentido prefiguran las ideas de trabajo con la comunidad, control social, la implicación en procesos institucionales y, por último, el concepto de vinculación y utilización del sistema de salud.
Participación como actividades con la comunidad. La gran mayoría de los entrevistados, y especialmente de las IPS privadas, describió la participación social en salud como una serie de actividades que las instituciones de salud llevan a cabo con la comunidad. Estas actividades son de promoción de la salud, prevención, reuniones sobre informes de funcionamiento de programas, actividades sociales de integración y motivación a los usuarios: «Es generar desde aquí actividades que pueden hacer un grupo de personas para un bien común, en este caso de la salud, para que se lleve a cabo todo lo que es la prestación de los servicios de salud» (Gerente de IPS privada). «Es tener actividades sociales con la comunidad y las IPS y EPS» (encargada de OAU de IPS privada).

Participación como control y seguimiento social a los servicios de salud. En las respuestas de algunos entrevistados (sobre todo personal de IPS privadas y algunos de las públicas), aparece el concepto de participación social en salud como una forma de control social y de seguimiento a la prestación de los servicios de salud: «...Aquél proceso o mecanismo donde la comunidad cuenta con un espacio para hacerle un seguimiento a los servicios de salud especialmente a aquellos que se denominan públicos» (Gerente IPS privada). «Es aquella en la cual todos los usuarios tienen la oportunidad de evaluar los servicios que se prestan en este momento y dar sugerencias para mejorar los servicios de la institución» (Gerente IPS pública).

Participación como la implicación de los usuarios en procesos institucionales. Solamente en el discurso de muy pocos gerentes de IPS públicas y de EPS privadas, se entiende la participación en salud como la oportunidad de los usuarios y la comunidad en general de participar en procesos institucionales para lograr el bien común, a través de mecanismos de participación definidos por las políticas, como la representación 
comunitaria en la junta directiva de las IPS. Es importante señalar que estos entrevistados hacen énfasis en que las instituciones permitan estas interacciones institucióncomunidad. «Participar en procesos que se realicen en las diferentes entidades. Obviamente se necesita que las diferentes entidades le permitan a los diferentes usuarios y a nuestros veedores participar de nuestros procesos...» (Gerente IPS pública). «Que una EPS tenga un representante de los usuarios ante la junta directiva de la EPS para que le pueda presentar la inconformidad que en un momento dado pueda tener un usuario» (Gerente EPS privada).

Participación como afiliación al sistema de salud. Parte del personal de salud, sobre todo de las EPS privadas y públicas, comparten la idea de que participación social en salud es estar afiliado al sistema de salud y tener derecho a utilizar los servicios de salud: "Es la oportunidad que tienen los usuarios o una población a tener unos servicios de salud y ser reconocidos en una institución...»(Gerente de EPS privada). «Siento $y$ creo que la participación es en este momento que la gente sepa la necesidad de estar afiliado a una entidad promotora de salud» (Encargada de OAU de EPS privada).

\section{Concepto de participación en salud de los formuladores de políticas}

Del discurso de los formuladores de políticas en salud, se deriva un núcleo de sentido que articula el trabajo comunitario organizado a un objetivo concreto como es la evaluación y control de los servicios de salud.

Participación como control social de los servicios de salud. Los formuladores de políticas definen el concepto de participación social articulado al sentido de evaluación y control social de los servicios de salud por la comunidad organizada, que debe llevar, según ellos, a que las instituciones pres- tadoras del servicio de salud lo presten con calidad. «La participación es la posibilidad de que los servicios sean mejor, que las diferentes organizaciones de usuarios analicen, discutan, participen en la prestación de servicios, que la gente lo califiquen bien regular o mal». (Secretario de Salud Municipal). «Para la Secretaría de Salud y sobre todo cuando está descentralizada, es importantísimo la participación más que todo en el control, la inspección, la vigilancia de estas instituciones prestadoras del servicio....» (Secretario de Salud Municipal).

\section{DISCUSIÓN}

Los resultados de ésta investigación permiten comprender, por una parte, el nivel de elaboración del concepto de participación en salud de los actores involucrados en la implementación de la política de participación en Colombia; y por otra, a develar los sentidos o significados construidos alrededor del concepto de participación social en salud que tienen estos mismos actores.

La participación en salud como construcción social, esta determinada por la dinámica social que le imprimen quienes interactúan en ese espacio, que son además quienes elaboran colectivamente y a partir de sus relaciones, los sentidos o significados que le otorgan. Se observó a partir del discurso de todos los entrevistados, distintos niveles de construcción del concepto de participación, así como de los distintos sentidos, que parecen reflejar sus propios roles y experiencias. Es decir que elaboran su noción de participación en salud, como se construye el saber popular: a partir de su experiencia y vivencia concreta, de sus interacciones con otros individuos o grupos ${ }^{16,26}$. Esto parece ser cierto, tanto para los conceptos expresados por los usuarios y líderes, como para los gerentes y encargados de las OAU de las de IPS y EPS, cuyos discursos se referían a experiencias concretas y directas de interacciones con los otros en lo cotidiano, y no estaban elabora- 
dos, como se habría podido esperar, a partir de los discursos técnicos de los documentos oficiales como leyes y decretos de salud, la Constitución etc. Este resultado en relación a este último grupo especialmente, parece estar reflejando una falta de conocimiento e información de las políticas de participación (o al menos de los núcleos temáticos principales de la norma) por parte de éstos actores, y, por tanto, estaría afectando la implantación de la política de participación social en salud en Colombia.

Por otra parte, la investigación intentó develar los sentidos que de participación en salud poseen distintos actores implicados en la implementación de la política en Colombia. Dicha política concedió gran importancia a la participación de los usuarios y de la comunidad, no sólo en aspectos puntuales, como proporcionar su opinión sobre los servicios de salud, sino ejerciendo su derecho de comunidad organizada de participar en todo el proceso relativo a los servicios de salud y sus programas (planeación, gestión y evaluación) y, sobre todo, consideró la participación en su más alto grado o nivel como es en la toma de decisiones sobre el sistema de salud (artículo 2 del decreto 1757 de 1994).

Los resultados del estudio parecen indicar que no hay una correspondencia de sentido entre el contenido explícito de la política y los diversos conceptos de participación que han construido cada uno de los diferentes actores involucrados en esta interacción. La conceptualización de la mayoría de los usuarios acerca de la participación, se exterioriza como un medio para expresar ideas u opiniones, lo cual indica que ellos mismos se ubican en un bajo nivel de participación -a nivel de consulta ${ }^{17,18}$. Resultados similares se encontraron en estudios recientes en Brasil ${ }^{14}$ y Colombia ${ }^{35}$.

La participación en salud también se entendió por parte de muchos usuarios, como estar dentro del sistema o hacer uso de los servicios de salud, que igualmente se circunscribe en el nivel más bajo de participación ${ }^{27}$. También el personal de salud conceptualizó la participación como la posibilidad de los usuarios de hacer uso de los servicios de salud, evidenciando que unos y otros se perciben en actividades y roles tradicionales, como consecuencia de su interacción cotidiana, como usuarios y como proveedores, El interaccionismo simbólico ${ }^{28}$ señala que nuestra condición humana deriva de los efectos mutuos entre los individuos, es decir, a partir de nuestras acciones e interacciones nos moldeamos y remoldeamos recíprocamente. Por tanto, en el contexto de la reforma del sector salud, donde los usuarios son percibidos como clientes que utilizan unos servicios y el personal de salud, como unos proveedores de los mismos $29,30,35$, es lógico que se configuren así estos actores en sus interacciones cotidianas de proveedor-usuario. Por otro lado, y como se propone en una reciente investigación en Brasil ${ }^{15}$, se debería considerar que entender la participación en salud como hacer uso de los servicios, también podría reflejar la necesidad no satisfecha de atención a la salud, debida la carencia de afiliación al sistema de salud de una parte importante de la población colombiana (aproximadamente $40 \%$ de la población no está afiliada al $\mathrm{SGSSS}^{36,37}$ ) que por tanto, sólo dispone de un acceso limitado a los servicios de salud.

Por otra parte, el concepto de participación en salud de buena parte de los líderes y usuarios mayores, articula sentidos de ayuda solidaria, voluntaria y de compromiso, y devela rasgos emergentes de una conciencia participativa anclada en una historia de construcción colectiva de poblaciones y de infraestructura de servicios públicos, así como de formas asociativas campesinas que están en la base de movimientos sociales del país de la mitad del siglo pasado hasta el presente. La solidaridad y el compromiso son valores sociales indispensables para la participación ${ }^{23,31,32,33}$. No obstante, no debe tratar de reducirse la participación al trabajo 
voluntario y solidario como mano de obra barata, gratuita y acrítica, sino que se deben considerar, la solidaridad y el compromiso, como valores que forman parte de los imaginarios colectivos y que pueden contribuir a catalizar comportamientos participativos, dentro de los procesos más ambiciosos de participación en la toma de decisiones respecto al sistema de salud.

Sólo se encontró en unos pocos actores, como los formuladores de políticas y algunos líderes y personal de salud, conceptos de participación articulados a la idea de control y seguimiento a los servicios de salud. Se encontró además, que sólo unos pocos gerentes de IPS públicas y privadas, así como algunos líderes, definieron la participación como un derecho de vinculación en procesos institucionales para poder influir en las decisiones, pero contando para ello, con estrategias que lo faciliten, como los mecanismos de participación institucionales o las organizaciones populares. Ambos sentidos son los que se acercan más a la concepción de participación en salud que contempla la norma y cuya limitada presencia denota, como se destaca más arriba, al menos el desconocimiento de la política.

Llama la atención que casi ninguno de los usuarios, a excepción de algunos líderes urbanos, se representó a la participación como un derecho o como un deber ${ }^{34}$, pero si como una actitud, voluntad y compromiso de implicación, conceptos estos, menos ajenos a su realidad cotidiana, y que estarían connotando un cierto fortalecimiento en su calidad de ciudadano y en un nuevo concepto de la participación como eje clave en la construcción de su destino.

Finalmente es necesario trabajar más en la difusión y conocimiento de las normas que regulan la participación en salud, capacitando tanto a líderes como al personal de salud, en el contenido, difusión y aplicación de las normas y mecanismos que regulan la participación social en salud. Esto permitiría, entre otros, que estos actores puedan establecer un diálogo y negociación social de ideas, conceptos y percepciones, a partir del núcleo central proporcionado por el artículo 103 de la constitución de Colombia, que define la participación en salud como «el proceso de interacción social para intervenir en las decisiones en salud, respondiendo a intereses individuales y colectivos en las decisiones de implantación, gestión, evaluación y veeduría en servicios de salud».

Por otra parte, se debe fomentar la participación de «abajo arriba», es decir, respetando la autonomía, el saber popular y la capacidad propositiva de las organizaciones populares $^{38}$, y, al mismo tiempo, trabajar en un cambio de percepción de la participación y del papel de los «otros» en dicha interacción social, puesto que la percepción es un proceso cognitivo mediador entre los pensamientos, intenciones y acciones, y no es exagerado decir que tan distintas percepciones sociales estarían obstaculizando la ejecución de la política participativa en Colombia. Finalmente, no se puede pretender que la población participe en procesos decisivos de un sistema de salud, al que no tiene acceso como usuario, y por tanto, el paso previo es garantizar el acceso a la atención en salud a los ciudadanos de acuerdo a su necesidad y no su capacidad adquisitiva.

\section{AGRADECIMIENTOS}

Estos resultados son parte de la investigación realizada en asociación con el Institute for Health Sector Development (Inglaterra), GEMA-Universidad del Valle (Colombia), Universidad Federal de Pernambuco y el Instituto Materno Infantil de Pernambuco (IMIP Brasil), EASP (España), con el apoyo del Consorci Hospitalari de Catalunya y las Secretarías de Salud de los municipios de Tuluá y Palmira y la Secretaría de Salud del Departamento del Valle del Cauca, en Colombia. Agradecemos a las personas entrevistadas en estos dos municipios que 
aceptaron compartir con los investigadores sus opiniones, ideas e inquietudes y a la Comisión Europea DG XXII por su ayuda financiera.

\section{BIBLIOGRAFÍA}

1. Decreto 1757. Por el cual se organizan y establecen las modalidades y formas de participación en la prestación de servicios de Salud. Bogotá, Colombia: Ministerio de Salud de Colombia, 1994.

2. Castro R, Hernández I. Opiniones de los diversos actores sobre la participaron comunitaria en salud y planificación familiar. Rev de Salud Pública de México1993; 35 (4):

3. Magalhaes ML, Bosi; K, de Carvalho Affonso. Cidadania, participação popular e saúde: com a palabra, os usuários da Rede Pública de Serviços. Cad Sáude Pública 1998; 14 (Sup 2): 59- 68.

4. Sánchez E. Participación comunitaria para la solución de problemas ambientales. Boletín de la AVEPSO 1996; 2: 18-26.

5. Mosquera M, Zapata Y, Lee K, Arango C, Varela A. Strengthening user participation through health sector reform in Colombia: a study of institutional change and social representation. Health Policy and Planning 2001; 16(2):52-60.

6. Castro, $\mathrm{R}$ y col. Relato de una experiencia de participación social en el distrito de Aguablanca de Cali, Colombia. Avances en Medicina Social. 1994; 14 (1): $1-22$.

7. Abrantes-Pêgo. Participación social en salud: un estudio de caso en Brasil. Salud Pública de México 1999; 41 (6): 466-474.

8. Zakus D y Lysack C. Revisiting community participation. Health Policy and Planning 1998; 13(1): 112

9. Brofman M. y Gleizer M. Participación comunitaria: ¿Necesidad, excusa o estrategia?. O ¿de qué hablamos cuando hablamos de participación comunitaria?. Cad Saúde Pública, 1994; 10 (1): 111-122.

10. Acioli MD, Carvalho E. Discursos e prácticas referentes ao proceso de participação comunitária nas ações de educação em saude: As ações de mobilização comunitária do PCDEN/PE. Caderno de Saúde Pública 1998; 14 (Sup 2): 59- 68.

11. Alves PC, Souza IM, Moura MA, Cunha LA. A Experiência da esquistossomose e os desafios da mobilização comunitária. Cad. Saúde Pública 1998; 14 (Sup.2): 79-90.

12. Álvarez JM. Corresponsabilidad y participación social. Salud Pública de México. 1992; 34: 74-81.

13. Organización Panamericana de la Salud. La participación social en el desarrollo de la salud. Serie Desarrollo y Fortalecimiento de los Sistemas Locales de Salud HSD/SILOS. Washington: OPS; 1992.

14. Vázquez ML. Ferreira MR. Siqueira E. Pereira AP. Diniz A. Leite I y Kruze I. Visión de los diferentes agentes Sociales sobre la participación social en el sistema de salud en el Nordeste de Brasil. Una Aproximación Cualitativa. Rev Esp de Salud Pública 2002; 76(5): 585-594.

15. Vázquez ML da Silva MRF. Siqueira E. Kruze I. Diniz A. Veras I \& Pereira A.P. Participação social nos serviços de saúde: concepções dos usuários e líderes comunitários em dois municípios do Nordeste do Brasil. Cad de Saúde Pública, Rió de Janeiro 2003; 19 (2): 109-118.

16. Valla, VV. Sobre participação popular: uma questão de perspectiva. Cad Saude Pública 1998; 14 (sup 2): 7-18.

17. Diaz Bordenave J. Participación y sociedad. Buenos Aires: Ediciones Búsqueda; 1985.

18. Gonzáles E. Manual sobre la participación y organización para la gestión local. Cali: Ediciones Foro Nacional por Colombia; 1996.

19. Ander-Egg E. Metodología práctica del desarrollo de la comunidad. México: Editorial el Ateneo. 1986.

20. Wolfe M. La Participación: una visión desde arriba. Argentina: CEPAL; 1984.

21. Sánchez M. Metodología y práctica de la participación. Madrid: Editorial Popular; 1986.

22. Rapapport J. Swift C, Hess R (eds). Studies in Empowerment. Binghamton, NY: Haworth; 1984.

Rev Esp Salud Pública 2005, Vol. 79, N. ${ }^{\circ} 6$ 
23. Viché M. Apuntes para la Participación Democrática desde el País Vasco Valenciano. En sociedad Civil e Instituciones Democráticas. Madrid: Editorial Popular; 1989

24. De Schutter A, Investigación participativa: una opción metodológica para la educación de adultos. Michoacán, México: CREFAL; 1986.

25. Ruiz Olabuénaga, JI. Metodología de la investigación cualitativa. Bilbao: Ed. Universidad de Deusto; 1999.

26 Fals Borda O. Conocimiento y poder popular. Bogotá: Siglo XXI Ediciones; 1986.

27. Vazquez ML. Expresión de Participacion social en los sistemas de salud de Latinoamérica. Proyecto de apoyo a la reforma del sector salud en Guatemala. Foro Internacional .La reforma del sector salud. Guatemala: 1999. p. 255-61.

28. Blumer H. Symbolic interactionism: perspective and method. NJ: Englewood Cliffs; Prentice-Hall Inc; 1969.

29. Klein R. The politics of de National Health Service. Harlow, UK: Longman; 1989.

30. Winkler F. Transferring power in Health Care. En: Dave B, Gary A, Seal C. (Eds). Health and Disease. A Reader. Second Edition. Milton Keynes, U.K: Open University Press; 1995.
31. Max Neef M. Desarrollo a escala humana. Medellín: Editores 20; 2000

32. Sarason S. The psychological sense of community. proaspects for a community psychology. San Francisco, CA: Jossey Bass; 1974.

33. Chavis D y Wandersman A. Sense of community in the urban environment: A catalyst for participation and community development. Am J Community Psychol 1990; 18: 55-81.

34. Vargas A. Participación social. una mirada crítica. Bogotá: Ed. Almudena; 2000.

35. Arévalo DA. Participación comunitaria y control social en el sistema de salud. Rev Salud Pública 2004; 6(2): 107-9.

36. De Groote T, De Paepe P, y Unger JP. Colombia: In vivo test of health sector privatization in the developing world. Int. Journal of Health Services 2005; 35(1):125-141.

37. Vargas I, Vázquez ML y Jané E. Equidad y reformas de los sistemas de salud en Latinoamérica. Caderno de Saúde Pública 2002; 18(4):927-937.

38. Delgado ME. Un modelo psicosocial como propuesta de análisis de las organizaciones populares. Congreso Regional de Psicología para Profesionales en América. IMIFAP/ AMEPSO/ IAAP. Entrelazando la Ciencia y la Práctica de la Psicología. Mexico 27 julio - 2 agosto de 1997. Libro de Memorias. 